\title{
Knee arthroscopy postoperative analgesia by multimodal cocktail regime
}

\author{
Ballal MM', Chebbi PK ${ }^{2}$, Prabhu $V^{3}$, Sathish Kimar $\mathrm{S}^{4}$, Chikkegowda $\mathrm{D}^{5}$ \\ ${ }^{1}$ Dr. Madan Mohan Ballal, ${ }^{2}$ Dr. Pavan Kumar Chebbi, ${ }^{3}$ Dr. Vasudev Prabhu, ${ }^{4}$ Dr. Sathish Kumar S, \\ ${ }^{5}$ Dr. Dharmesh Chikkegowda.
}

Address of Correspondence: Dr. Madan Mohan Ballal- madanballal@gmail.com, Dr. Pavan kumar Chebbipavanchebbi267@yahoo.co.in

\begin{abstract}
Background: Post-operative knee pain management have become a challenge to provide early relief and pain free postoperative care to the patient. Adequate analgesia influences the early rehabilitation of the patient and hospital stay. Many factors have been implicated to influence post arthroscopy pain such anesthetic technique, residual effects of perioperative analgesia, patient pain threshold, preoperative pain level, the use and duration of tourniquet exsanguination, surgical trauma involved, volume of drug injected, the experience of the surgeons, the sex of the patient and the postoperative activity level of the patients. The purpose of this study is to assess the efficacy of the multidrug regime for management of postoperative pain during first 24 hours. Methods: In our study 57 patients of both the sexes were evaluated for postoperative pain following various elective arthroscopic knee surgeries such as ligament reconstruction, cartilage procedures and diagnostic arthroscopic procedures. Patients were evaluated for pain using visual analogue score at $6^{\text {th }}$ hour, $12^{\text {th }}$ hour and 24 hours' post-operative. A cocktail prepared of multidrug was used for the study. Results: The new cocktail regime provides adequate analgesia with no patients requiring additional analgesia for first 12 hours and only 2 patients whose VAS [visual analogue scale] was greater than 4 required additional analgesia at the end of 24 hours postoperative. Conclusion: Multi drug cocktail regime provides a good analgesic for post-operative knee arthroscopic surgery without the need of rescue analgesics. Intra articular local analgesia reduces overall use of parenteral analgesics and also helps in quicker rehabilitation.
\end{abstract}

Keywords: Post-operative analgesia, Post arthroscopy analgesia, Intraarticularanalgesia, Cocktailanalgesia, Knee arthroscopy

\section{Introduction}

Arthroscopic knee surgery has become increasingly popular in the modern orthopaedics. Post-operative knee pain management has become a challenge to provide early relief and pain free postoperative care to the patient. Pain management has become a priority for management as a day care procedure. Patients operated as a day care procedure should have minimal postoperative pain which influences the timing of discharge. Pain is due to multifactorial causes such as irritation of free nerve endings of joint capsule, synovialtissue, anterior fat pad [1]. In an effort to provide an effective, safe and long lasting post arthroscopy analgesia, several studies using different

Manuscript received $4^{\text {th }}$ May 2016

Reviewed: $14^{\text {th }}$ May 2016

Author Corrected: $26^{\text {th }}$ May 2016

Accepted for Publication $18^{\text {th }}$ June 2016 drugs and regimes have been tried. Traditionally, oral analgesics are prescribed for the management of postoperative pain. The routine use of oral opioid analgesics administered on an frequently results in inadequate pain relief as they are not site specific and possess additional side effects such as nausea and vomiting. Manyfactors have been implicated to influence post arthroscopypain such anesthetic technique, residual effects of perioperative analgesia, patient pain threshold, the use and duration of tourniquet exsanguination, surgical trauma involved, volume of drug injected, the experience of the surgeons, the sex of the patient and the postoperative activity level of the patients. Post-operative pain levels are also influenced by the preoperative pain levels of the patient $[2,3,4,5,6,7,8]$. The purpose of anesthesia is not only 
the reduction of pain but to prevent it. Pain after a knee arthroscopy is found to be higher during first 8 hours and reaching a plateau at the end of 24 hours, requiring multiple doses of additional analgesia as there is not a single dose drug which can provide adequate analgesia for 24 hours. The purpose of this study is to assess the efficacy of the multidrug regime for management of postoperative pain during first 24 hours.

\section{Materials and Methods}

In our study 57 patients of both the sexes were evaluated for postoperative pain following various elective arthroscopic knee surgeries such as ligament reconstruction, cartilage procedures and diagnostic arthroscopic procedures. Patient's age, sex and weight were noted. Patient was explained regarding the surgical procedure and the study prior to the surgery. Patients who had additional comorbid conditions were not include in the study. All the surgeries were done by the same surgeon. Patients were evaluated for pain using visual analogue scale at $6^{\text {th }}$ hour, $12^{\text {th }}$ hour and 24 hours'postoperative. In this study a new cocktail of multiple drugs was prepared at our institute. The new cocktail regime prepared consistsof multiple analgesics to reduce post-operative pain after knee arthroscopy.

Cocktail mixture consists of

1. Ropivacaine $0.75 \mathrm{mg}(20 \mathrm{ml})$

2. Ketorolac 1 ampule

3. Morphine $5 \mathrm{mg}$

4. Clonadine half ampule

5. 1 ampule Adrenaline in $10 \mathrm{ml}$ saline-Take $1 \mathrm{ml}$ of this mixture

Mix this into saline to make it $50 \mathrm{ml}$

All the patients were informed about the procedure preoperatively and VAS [0- NO PAIN, 10-WORST PAIN] was explained to them. Patients were enquired about any drug allergy and all relevant investigations needed for the surgery was done and their results were noted. Minimum period of 6 hours of fasting was followed before the surgery. All the patients received tablet diazepam $10 \mathrm{mg}$ orally to relieve anxiety and sound sleep. On entering the patient in the operative room, standard intraoperative monitors like ECG, pulse oxymeter, non-invasive blood pressure were attached and baseline parameters were recorded. All the patients were operated after spinal anesthesia was administered. Tourniquet was used to minimize blood loss and to provide a clear view of the operating area. Pneumatic tourniquet was used with pressure of $300 \mathrm{mmHg}$ after leg is raised for 4 minutes. Cocktail mixture was freshly prepared during the surgery and administered at the end of the surgery through the suprapatellar pouch into intraarticular site. Tourniquet was deflated 10 minutes after the administration of analgesia. Patients were evaluated for pain at 6th hour, 12th hour and at the end of 24 hourspost-operative and the pain was assessed by VAS [9]. VAS was used for assessment of pain as it is simple and efficient. VAS can be easily used on sick and poorly educated patients who can grasp the nature of the scale without difficulty. Any patient with VAS greater than 4 requiring rescue analgesics were noted. The occurrence of side effects such as hypotension, bradycardia, nausea,vomiting,sedation was recorded simultaneously while assessing VAS.

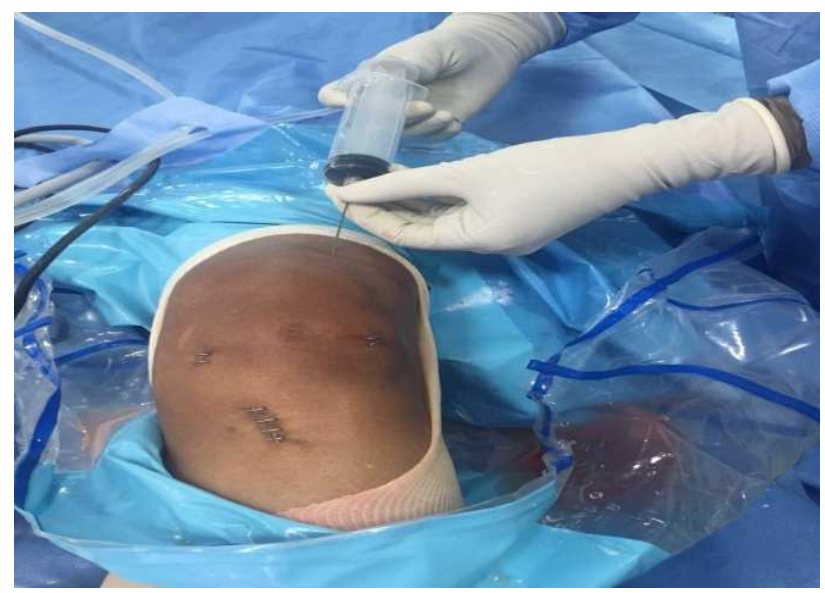

Figure-1: Intraarticular cocktail administration through the suprapatellar pouch

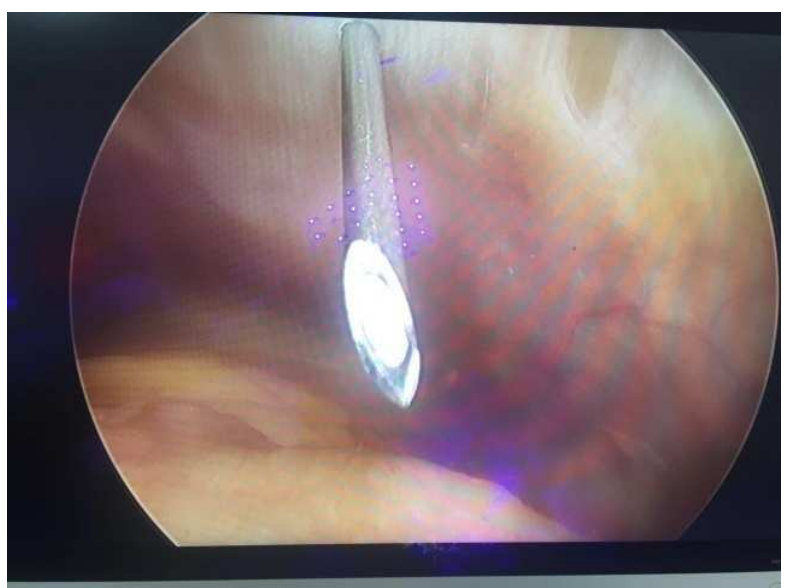

Figure-2: Arthroscopic confirmation of the needle placement 


\section{Results}

Table 1: showing VAS score at $6,12 \& 24$ hours post operatively.

\begin{tabular}{|c|c|c|c|}
\hline VAS Score & 6 hours post operative & 12 hour post operative & 24 hours post operative \\
\hline VAS 0 & 49 & 40 & 34 \\
\hline VAS 2 & 6 & 10 & 11 \\
\hline VAS 4 & 2 & 7 & 10 \\
\hline
\end{tabular}

Table showing VAS score at $6,12 \& 24$ hours post operatively. After 6 hours almost 49 patients have score 0 . At the end of 24 hours 34 patients have scored $0 \& 11$ have scored 2.

\section{Discussion}

Arthroscopic knee surgeries are associated with invariable amount of pain post operative causing patient discomfort and prolong hospital stay. Compared to other modalities of pain management, intraarticular route provides a continuous and stable pain management for first 24 hours post operative. Good duration of analgesia and decreased need of analgesics post operatively is obtained through intraarticular route due to poor absorption caused by vascular disruption at the operated site. Patients had a reduced incidence of nausea and vomiting due to decreased systemic absorption. No side effects related to analgesic mixture were reported during the period of study. Cardiovascular parameters didnot show any significance changes compared to presurgical conditions. The aim of the study was to assess the postoperative pain during the first 24 hours. All the patients included in the study were admitted in the hospital for a minimum period of 24 hours so that the patient's pain could be assessed and adequate rescue analgesia could be administered.

Ropivacaineis a local anesthetic used commonly. It is lipid soluble and provides a effective post-operative analgesic [10]. The use of intraarticular ropivacaine provides improved levels of post operative analgesics after knee surgery as compared with bupivacaine. The safety margin of rupivacaine is also well tolerated by many patients [11]. Lidocaine is a local anesthetic and has analgesic, antihyperalgesic and anti-inflammatory properties. Mechanism of action of lidocaine is mainly due to blockade of sodium channels and of N-methyl D-aspartate and $G$ protein coupled receptors [12, 13]. Lidocaine anti-inflammatory property is by reducing the levels of cytokines by inhibiting neutrophil activation. It is also known to cause reduction in pro and antiinflammatory cytokines and suppression of lymphocyte proliferation which influences acute phase inflammatory reactions $[14,15]$. Studies have shown that local anesthetic are chondrotoxic in vitro studies. Chondrolysis was mainly observed when used on a continuous dosage. Even single dose of bupivacaine was found to cause late cellular and metabolic changes in the chondrocytes in animal studies [16]. Meanwhile single intra-articular injection of lidocaine hydrochloride used for knee arthroscopy do not influence the viability, morphology, and cultivation potential of chondrocytes in articular cartilage biopsy specimens assigned for autologous chondrocyte implantation [17].

Ketorolac is a non-steroidal anti-inflammatory drug (NSAID) used as ananalgesic. Ketorolac reduces the local concentration of allogenic concentration, thereby reducing the sensitivity of peripheral nociceptors which are activated by tissue injury. Ketorolac have been shown to give superior post arthroscopy analgesia when administered intraarticularly rather than intravenously. Administrations of intra articular ketorolac further reduce inflammation on the surface of the knee joint, and thus improve analgesia when combined with bupivacaine [18].

Clonidine may act on presynaptic $\alpha$ adrenergic receptors and inhibit the release of norepinephrine atperipheral afferent nociceptors. Clonidine has also been shown to provide a local anesthetic effect which inhibits the conduction of nerve signals through $\mathrm{C}$ and 
Affibers [19]. Morphine is a Opioid which can produce potent anti-nociceptive effects by interacting with local opioid receptors in inflamed peripheral tissue. Low doses of intraarticular morphine can significantly reduce pain after knee surgery through an action specific to local opioid receptors that reaches its maximal effect three to six hours after injection [20].

Many studies have been conducted to assess the analgesic effects of morphine. Most of the consistent results as good analgesics have been obtained when morphine was used through intrarticular route. The results of the study showed that morphine when used intraarticularly had better control of pain compared to other analgesics while in some study no difference was noted [21, 22]. Several studies have shown that higher concentration of opioids in a lower volumes increases the speed of onset of analgesia [23, 24].

The analgesic effect of morphine when administered intra -articulary has shown to last around 48 hours but further study is required to know the mechanism of prolonged analgesia [25].

Duration between the administration of intra - articular analgesic and tourniquet plays a significant role. Adequate time should be provided between administration of analgesics and tourniquet release leads to increase in the binding of the analgesics to the inarticular tissue providing optimum analgesia [26]. In our study a minimum duration of 10 minutes was provided between the administration of analgesics and tourniquet release.

Preoperative pain levels of the patients also seem to influence the post-operative pain levels. Patients with middle preoperative pain levels had a higher amount of post operative pain levels compared to patients with low pre-operative pain level. While the patients of high preoperative pain levels had a mixed outcome [27]. So patient in the study levels should have similar levels of pain to avoid its influence on the study. Though the study was primarily done for the assessment of analgesic effect of the new cocktail regime, there were certain short comings of the study.

Blood levels of the various drugs were not tested to analyse the relation between the blood concentration and analgesia effect. The degenerative changes of the cartilage could not be assessed as the long term follow up of the patient was not carried out. From the study only the short term results only could be assessed long term effects could not be assessed.

\section{Conclusion}

Multi drug cocktail regime provides a good analgesic for post-operative knee arthroscopic surgery without the need of rescue analgesics. Intra articular local analgesia reduces overall use of parenteral analgesics and also helps in quicker rehabilitation.

Funding: Nil, Conflict of interest: None initiated. Permission from IRB: Yes

\section{References}

1. Dye SF, Vaupel GL, Dye CC. Conscious neurosensory mapping of the internal structures of the human knee without intraarticular anesthesia. Am J Sports Med. 1998 Nov-Dec; 26(6):773-7.

2. Henderson RC, Campion ER, DeMasi RA, Taft TN. Post arthroscopy analgesia with bupivacaine. A prospective, randomized, blinded evaluation. Am J Sports Med. 1990 Nov-Dec;18(6):614-7.

3. Laurent SC, Nolan JP, Pozo JL, Jones CJ. Addition of morphine to intra-articular bupivacaine does not improve analgesia after day-case arthroscopy. Br J Anaesth. 1994 Feb;72(2):170-3.

4. Johnson DS, Stewart H, Hirst P, Harper NJ. Is tourniquet use necessary for knee arthroscopy? Arthroscopy. 2000 Sep;16(6):648-51.

5. Kirkley A, Rampersaud R, Griffin S, Amendola A, Litchfield R, Fowler P. Tourniquet versus no tourniquet use in routine knee arthroscopy : A prospective, double blind, randomised clinical trial. Arthroscopy. 2000; 16(2):121-6.

6. Rosseland LA, Stubhaug A, Skoglund A, Breivik H. Intra-articular morphine for pain relief after knee arthroscopy. Acta Anaesthesiol Scand. 1999 Mar; 43(3): 252-7.

7. Rosseland LA, Stubhaug A. Gender is a confounding factor in pain trials: women report more pain than men after arthroscopic surgery.Pain.2004Dec;112(3):248-53.

8. Whitford A, Healy M, Joshi GP, McCarroll SM, O'Brein TM. The effect of tourniquet release time on the analgesic efficacy of intraarticular morphine after arthroscopic knee surgery. Anesthesia and Analgesia. 1997;84(4):791-3.

9. Chapman CR, Casey KL, Dubner R, Foley KM, Gracely RH, Reading AE. Pain measurement: an overview. Pain. 1985 May;22(1):1-31. 
10. Kaeding CC, Hill JA, Katz J, Benson L. Bupivacaine use after knee arthroscopy: pharmacokinetics and pain control study. Arthroscopy. 1990; $6(1): 33-9$

11. Emmanuel Marret, Marc Gentili, Marie Pierre Bonnet, Francis Bonnet. Intra-articular ropivacaine $0.75 \%$ and bupivacaine $0.50 \%$ for analgesia after arthroscopic knee surgery: A randomized prospective study. Presented at the Euroanaesthesia meeting, Glasgow, Scotland, May 2003.DOI: http://dx. doi. org/10.1016/j.arthro.2004.11.005

12. Koppert W, Ostermeier N, Sittl R, Weidner C, Schmelz M. Low-dose lidocaine reduces secondary hyperalgesia by a central mode of action. Pain. 2000 Mar; 85(1-2):217-24.

13. Hollmann MW, Durieux ME. Local anesthetics and the inflammatory response: a new therapeutic indication? Anesthesiology. 2000 Sep;93(3):858-75.

14. MacGregor RR. The effect of anti-inflammatory agents and inflammation on granulocyte adherence. Evidence for regulation by plasma factors.Am J Med. 1976 Nov;61(5):597-607.

15. Mac Gregor RR, ThornerRE, Wright DM. Lidocaine inhibits granulocyte adherence and prevents granulocyte delivery to inflammatory sites. Blood. 1980;56(2):203-9.

16. Piper SL, Kramer JD, Kim HT, Feeley BT. Effects of local anesthetics on articular cartilage. Am J Sports Med. 2011 Oct;39(10):2245-53. doi: 10.1177/ 0363546511402780. Epub 2011 Apr 22.

17. Ravnihar K, Barlič A, Drobnič M. Effect of intraarticular local anesthesia on articular cartilage in the knee.Arthroscopy. 2014 May;30(5):607-12. doi: 10. 1016/j.arthro.2014.02.002.

18. Reuben SS, Connelly NR. Postoperative analgesia for outpatient arthroscopic knee sugery with intraarticular bupivacaine and ketorolac. AnesthAnalg. 1995 Jun; 80(6):1154-7.
19. Butterworth JF 5th, Strichartz GR. The alpha 2adrenergic agonists clonidine and guanfacine produce tonic and phasic block of conduction in rat sciatic nerve fibers. Anesth Analg. 1993 Feb;76(2):295-301.

20. Stein C, Comisel K, Haimerl E, Yassouridis A, Lehrberger K, Herz A, Peter K. Analgesic effect of intraarticular morphine after arthroscopic knee surgery. N Engl J Med. 1991 Oct 17;325(16):1123-6.

21. Kalso E, Tramèr MR, Carroll D, McQuay HJ, Moore RA. Pain relief from intra-articular morphine after knee surgery: a qualitative systematic review. Pain. 1997 Jun;71(2):127-34.

22. Stein C, Schafer M, Cabot PJ, etal. Peripheral opiodanalgesia. Pain Rev 1997;4:173-87.

23. Stein C, Comisel K, Haimerl E, Yassouridis A, Lehrberger K, Herz A, Peter K. Analgesic effect of intraarticular morphine after arthroscopic knee surgery. N Engl J Med. 1991 Oct 17;325(16):1123-6.

24. Joshi GP, McCarroll SM, Cooney CM, Blunnie WP, O'Brien TM, Lawrence AJ. Intra-articular morphine for pain relief after knee arthroscopy. J Bone Joint Surg Br. 1992 Sep;74(5):749-51.

25. Khoury GF, Chen AC, Garland DE, Stein C. Intraarticular morphine, bupivacaine, and morphine/ bupivacaine for pain control after knee videoarthroscopy. Anesthesiology. 1992 Aug;77(2):263-6.

26. Katz JA, Kaeding CS, Hill JR, Henthorn TK. The pharmacokinetics of bupivacaine when injected intraarticularly after knee arthroscopy. AnesthAnalg. 1988 Sep;67(9):872-5.

27. Drosos GI, Stavropoulos NI, Katsis A, Kesidis K, Kazakos K, Verettas DA. Post-operative pain after knee arthroscopy and related factors. Open Orthop J. 2008 Jun 13;2:110-4. doi: 10.2174/1874325000802010110.

\section{How to cite this article?}

Ballal MM, Chebbi PK, Prabhu V, Sathish Kimar S, Chikkegowda D. Knee arthroscopy postoperative analgesia by multimodal cocktail regime. Int J Med Res Rev 2016;4 (7):1120-1124.doi: 10.17511/ijmrr.2016.i07.09. 